development of brain structures and function. We studied the participation of proteins having neurotrophic and neurogrowth activities, like S100beta and A3G7, in the adult rat model of short- and long-term habituation of acoustic startle reaction (ASR) with freezing behaviour recorded simultaneously. It was observed that rabbit polyclonal antibodies against protein S100beta, and monoclonal antibodies against protein A3G7, influenced in a selective and a dose-dependent way both storage and retrieval of ASR. Application of antibodies against S100beta and A3G7 in various brain structures (cerebellar cortex, hippocampus) caused different types of behavioural effects. Subsequently, the increase of S100beta ratio in several brain structures (basal nuclei, frontal cortex, hippocampus, hypothalamus, cerebellum) was determined by ELISA method at various delays $(0,0,5,1,2,4,6,8,24$ and 48 hours) following elaboration of long-term habituation of ASR in adult rats. We found that S100beta content in each structure showed specific temporal and quantitative variations. The present data provide experimental evidence of the heterochronia of molecular mechanisms associated with learning and memory in the adult brain.

Supported by Russian Foundation for Basic Research, grant \#8470 97-04-49092, and Federal Program "State support for high education and fundamental science integration 1997-2000" (grant 313).

\title{
Learning and Memory: Genetics
}

\section{LMG1}

GENETIC DIFFERENCES IN RESPONSE TO NOVELTY AND SPATIAL MEMORY: STUDY IN A TWO-TRIAL RECOGNITION TASK IN MICE

F. Dellu', A. Contarino', H. Simon', G.F. Koob ${ }^{1}$, and L. Gold ${ }^{1}$

${ }^{I}$ The Scripps Research Institute, La Jolla, USA and ${ }^{2}$ Laboratoire de Neuropsychobiologie des Désadaptations, CNRS UMR 5541, Bordeaux, France

A two-trial memory task, based on a free-choice exploration paradigm in a Y-maze, was previously developed to study recognition processes in Sprague-Dawley rats (Dellu et al., 1992 ; 97). Because this paradigm avoids the use of electric shock or deprivation that may have non-specific effects and does not require learning of a rule, it may be particularly useful for studying memory in mice. Four inbred strains (Balb/cByJ, DBA/2J, C57BL/6J, and SJL/J), an F1 Hybrid (C57BL/6 x SJL/J), and one outbred strain (CD1) were used to validate this task in mice and to characterize a strain distribution in response to novelty and working memory. During the first trial (acquisition), the animal is allowed to visit 2 arms of a Y-maze, the third being blocked with a door. During the second trial (retrieval), the door is opened, and the animal has free access to all three arms. Discrimination of novelty versus familiarity can then be studied by comparing exploration of the 3 arms. Exploration was measured with a short ( $2 \mathrm{~min}$ ) inter-trial interval (ITI) between acquisition and retrieval, while memory was examined with longer intervals ( 30 min, $1 \mathrm{~h}, 2 \mathrm{~h}$ ). A study of the time course of the response to novelty revealed varying degrees of preference and/or habituation to novelty among the differer.t strains with CD1 exhibiting a very high response to 
novelty and others showing lower (C57 x SJL hybrids) to complete absence (SJL) of exploration of novelty. Memory span, assessed with increasing ITIs, varied widely among strains from 2 min (C57 x SJL hybrids), $30 \mathrm{~min}$ (DBA), $1 \mathrm{~h}$ (CD1) to at least $2 \mathrm{~h}$ (C57 and BALB). No relationship between mnesic performances and locomotor activity nor response to novelty could be discerned. Such demonstrated sensitivity to a wide range of behavioral phenotypes supports the use of this spatial memory task as an effective tool for the study of genetic influences on the response to novelty and recognition processes in mice.

\section{LMG2}

EXCITOTOXIC HIPPOCAMPAL LESIONS IN MICE: EFFECTS OF STRAIN AND TASK DEMANDS ON SPATIAL DISCRIMINATION

M. Arns ${ }^{l, 2 *}$, M. Sauvage ${ }^{l}$ and T. Steckler ${ }^{l}$

${ }^{\prime}$ Max Planck Institute of Psychiatry, Kraepelinstraße 2 - 10, D-80804 Munich, Germany, ${ }^{2}$ Deptartment of Comparative and Physiological Psychology, University of Nijmegen, Nijmegen, The Netherlands

Spatial discrimination learning of ibotenic acid-lesioned C57BL/6 (B6) and DBA/2 (D2) mice was tested in two-choice water maze and cross maze tasks. In the water maze spatial discrimination, two visible, identically looking platforms were used, one being stable and providing support (correct), the other sinking when a mouse tried to climb on it (incorrect). On each trial, the correct platform remained in the same spatial position, while the incorrect platform and starting positions changed from trial to trial. During acquisition (6 sessions, 10 trials per session), B6 but not D2 mice improved choice accuracy. Furthermore, choice latency and the distance travelled to reach the platforms were longer in mice of the D2 strain which also swum at lower speed compared to B6 mice. Hippocampal lesions impaired accuracy in mice of the B6 strain. In lesioned mice from both strains, errors of omission, choice latency, distance to reach the platforms and thigmotactic behaviour, but not speed, were increased during this experimental stage. Relocation of the correct platform position, however, indicated that B6 hippocampus-lesioned animals had acquired some knowledge about its previous position. In the cross maze, animals had to choose between two maze arms, both being in stable positions relativ to distant spatial cues, but only one containing a food pellet, while their starting position varied from trial to trial. In contrast to the water maze task, accuracy did not differ between strains, but there was an overall effect of lesion. Furthermore, D2 hippocampus-lesioned animals developed a strong side bias over sessions. Thus, ibotenic acidinduced lesions of the hippocampus cause spatial memory impairments in mice. These lesion-induced deficits were strain-dependent and likely comprise of impaired accuracy as well as changes in nonmnemonic types of behaviour. Importantly, lesions in both strains impaired spatial learning, and whether a deficit was seen in hippocampal animals of the D2 strain seems to depend on the demands of the task. 
LMG3

COGNITIVE IMPAIRMENTS AND HYPEREXPLORATION OF OBJECTS IN RATS SELECTIVELY BRED FOR AUDIOGENIC SEIZURE SUSCEPTIBILITY

M.G. Pleskacheva ${ }^{l}$, Z.A. Zorina ${ }^{l}$, D.P. Wolfer ${ }^{2}$, and H.-P. Lipp ${ }^{2}$

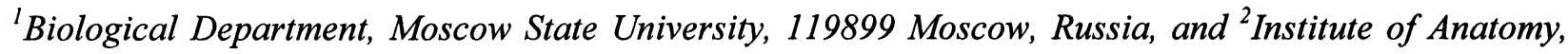
University of Zurich, CH-8057 Zurich, Switzerland

Krushinsky-Molodkina albino rats (KM) have been selectively bred for audiogenic seizure susceptibility and show clonic and tonic seizures to the sound of a electric bell (Krushinsky, 1962; Krushinsky et al., 1970). They were compared here with normal albino rats on three different tests. An open field test (arena with five objects) revealed that a majority of KM rats visited object zones more frequently than Wistar rats and showed hyperactive object exploration, grooming, staying closely to objects, and high levels of defecation. Habituation of exploratory activity in the course of daily sessions was slow in these KM rats. A minority of KM rats (about $25 \%$ ) showed very low activity, no exploration and strong freezing. The cognitive tests revealed learning and memory deficits in KM rats. They showed slow acquisition in Morris water maze both in the spatial and in the cued version, a less effective strategy of goal platform searching, and high scores of floating and thigmotaxis. However, no significant strain differences as compared to Wistar rats were found during the probe trial in the spatial variant of the Morris test. Another test based on the localisation of a shifting food bait (the Revecz-Krushinsky task) showed that KM rats have difficulties in remembering the exact location of the shifting bait. These results are in agreement with reports of possible deficits in working memory in KM rats (Batuev, Ryabinskaya, Ashihmina, 1983). Thus KM rats appear to have hippocampal deficits in spite of the fact that the origin of the audiogenic seizures is subcortical and that the anatomy of the hippocampus appears to be normal.

Supported by RFFI 98-04-48440, SNF 7IP051224 and SNF 31-46691.96.

\section{LMG4}

\section{GDNF HETEROZYGOUS MUTANT MICE IN TWO GENETIC BACKGROUNDS EXHIBIT SPATIAL TASK SPECIFIC COGNITIVE DEFICITS IN THE WATER MAZE}

R. Gerlai*, D. Choi-Lundberg, J. Ross, L. Powell-Braxton, and H.S. Phillips

Neuroscience \& Cardiovascular Depts., Genentech Inc., 1 DNA Way, South San Francisco, CA 94080, USA

Glial cell line-derived neurotrophic factor (GDNF) is a potent neurotrophic factor for dopaminergic neurons in the mammalian brain. Research has been focused on brain areas (e.g. substantia nigra, striatum, and nucleus accumbens) where dopaminergic neurons play crucial role and perhaps represent therapeutic targets for alleviating Parkinsonian symptoms. Recent studies, however, also showed that GDNF and their receptors, GFRa1, and c-Ret (the signaling receptor) are all expressed in the mammalian 
hippocampus, a brain area playing a central role in learning and memory. Furthermore, electric or ischemic stimulation induced transcription level changes in GDNF and its receptors have been reported in the hippocampus, and anecdotal evidence exists on systemic GDNF replacement therapy in humans leading to cognitive disturbances. Here we analyze heterozygous mice with a null mutation in GDNF. Pathologic and hematologic analyses showed that mutant mice do not suffer from gross abnormalities. Neurochemical analysis and tests of amphetamine induced locomotory behavior suggested that mutant mice possess an intact dopaminergic system. Interestingly, however, mutant mice exhibited a significant impairment in the spatial version of the Morris water maze, a task sensitive to hippocampal dysfunction. General performance factors, e.g. swimming speed or non-spatial learning, were unaltered. The results were replicated in two genetic backgrounds, a 129SV x C57BL/6 F2 hybrid and a C57BL/6 backcross (ninth generation) suggesting that the alteration is caused by the null allele. These results demonstrate that GDNF plays an important role in cognition associated with hippocampal function. They, together with previously published results, also raise the possibility that GDNF and its receptors may represent an important therapeutic target in epilepsy, ischemic brain insult, or neurodegeneration in the adult mammalian hippocampus.

\section{LMG5}

ROLE OF TISSUE PLASMINOGEN ACTIVATOR IN MUTIPLE FORMS OF SYNAPTIC PLASTICITY AND MEMORY-RELATED BEHAVIOURS

L. Restivo ${ }^{I, 2}$ * N. Berretta ${ }^{2}$, P. Gubellini ${ }^{2}$, A. Gulino ${ }^{2}$, P. Calabresi ${ }^{2}$, and M. Ammassari-Teule ${ }^{I, 2}$

${ }^{\prime}$ Istituto di Psicobiologia e Psicofarmacologia, Consiglio Nazionale delle Ricerche and ${ }^{2}$ IRCCS S. Lucia, Rome, Italy

Tissue plasminogen activator (tPA) is a serine protease involved in tissue remodelling and, more generally, in the modulation of the neural architecture. Its role in learning and memory has also been demonstrated since mice lacking the gene encoding for t-PA show both reduced late phase of hippocampal LTP and mild learning deficits. In order to better characterise the role of t-PA in the control of memory-related behaviours, the present experiments have compared the ability of t-PA-KO and wild type (WT) mice in a number of hippocampal- and non hippocampal-dependent tasks. Mice were first examined in a situation of object exploration followed by a test of reactivity to spatial change and to object change. Subsequently they were tested for contextual and cue fear conditioning. Finally, they were subjected to a five day-active avoidance training. In general, the behaviour of t-PA-KO mice was found to reflect both an impaired hippocampal and striatal function. That is, t-PA-KO showed deficits in contextual fear conditioning, habituation of object exploration and reactivity to spatial change, like animals with a hippocampal dysfunction. However, $\mathrm{t}-\mathrm{PA}-\mathrm{KO}$ also displayed poor rearing activity and active avoidance performance, like animals with a striatal dysfunction. In agreement with these behavioural data, (i) high frequency cortical stimulation was found to induce a more consistent striatal LTD in WT than in t-PA-KO and (ii) tetanic stimulation of Schaeffer collateral-commissural fibres 
produced a significant post-tetanic potentiation of the excitatory transmission in the CA1 area of the hippocampus in both groups, but this activity declined more rapidly in t-PA than in WT mice. Taken together, these data indicates that t-PA plays a critical role in various forms of synaptic plasticity and memory-related behaviours.

\section{LMG6}

IMPROVED SPATIAL LEARNING OF APOLIPOPROTEIN E KNOCKOUT MICE FOLLOWING REPEATED EXPOSURE TO RATS: INVOLVEMENT OF CELL ADHESION MOLECULES ?

J. Grootendorst ${ }^{\prime *}$, M.S. Oitzl ${ }^{l}$, S. Dalm ${ }^{\prime}$, E.R. de Kloet ${ }^{l}$, and C. Sandi ${ }^{2}$

${ }^{\prime} L A C D R$, Division of Medical Pharmacology, University of Leiden, P.O Box 9503, 2300 RA Leiden and

${ }^{2} U N E D$, Division of Psychobiology, Ciudad Universitaria s/n, 28040 Madrid, Spain

Because apolipoprotein E4 (apoE4) has been identified as a risk factor for Alzheimer's disease, a role for apoE in synaptic plasticity and cognition has been suggested. Previously, we have shown that apoEknockout mice are indeed impaired in spatial learning and memory (Oitzl et al. (1997) Brain Res.752:189) and show altered synaptic plasticity in hippocampal neurones (Krugers et al. (1997) NeuroRep. 8:2505). Recently we found that prior exposure of wild type mice to a stress paradigm impaired spatial learning, but to our surprise the apoE-knockouts showed improved cognitive performance (Grootendorst et al. (1998) Soc. Neurosci. Abst 24:670.5). To address this issue we focused on cell adhesion molecules (CAM's), because CAM's mediate the cognitive effects of corticosteroid hormones secreted after stress (Sandi (1998) Neural Plasticity 6 (30):41). To further explore the mechanism underlying the strikingly opposed outcome of the stress effect on memory performance of apoE knockouts and wild type mice, we investigate how CAM's are implicated. For this purpose we have applied the same stress paradigm as previously used (i.e exposure of mice to rats). At three hours after the last training trial in the water maze, mice were decapitated and brains were dissected into the following regions: cerebellum, frontal cortex, hippocampus, cortex. The expression of NCAM and L1 was measured by ELISA. Under basal conditions, a differential regulation of NCAM and L1 in apoEknockout mice compared to wild type mice was found. In apoE-knockout mice, NCAM was decreased in the frontal cortex, whereas L1 was increased in frontal cortex and hippocampus compared to wild type mice. These observations underscore an important role of cell adhesion molecules in mediating steroid effects on cognition against apoE genetic background, and we hypothesise dynamic changes in CAM expression during cognitive performance of these mice as well.

Supported by ISAO 679756-0270, NWO 970-10-007 and EC BiotecPL960179. 


\section{LMG7}

\section{REACTION TO NOVELTY AND SPATIAL ORIENTATION IN ALPHA1B(-/-) KNOCKOUT MICE}

M. Spreng ${ }^{\prime *}$, S. Cotecchid ${ }^{2}$, and F. Schenk ${ }^{l}$

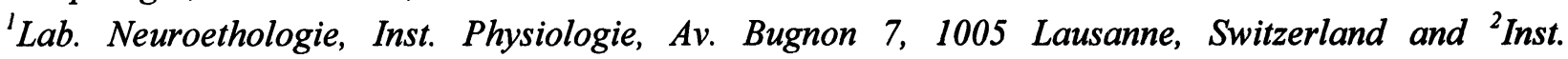
Pharmacologie, Av. Bugnon 7, 1005 Lausanne, Switzerland

Knockout mice lacking alphalb noradrenergic receptors were tested in behavioural experiments to test a possible effect of the absence of this receptor in reaction to novelty and spatial orientation. Reaction to novelty was tested in two experiments. In the first one the mice' latency to exit the first part of a two compartment set-up was measured. The knockout mice were faster to emerge then their littermate controls. Then they were tested in an open-field, in which new objects were added at the second trial. In the open-field without objects (first trial), the knockout mice showed a greater locomotor activity (path length). Then the same mice showed enhanced exploration of the newly introduced objects, relative to the control. The spatial orientation experiments were done on a homing board and in the water maze. The homing board did not yield a significant difference between the knock-out and the control mice. Both groups showed impaired results when the proximal (olfactory) and distal (visual) cues were disrupted by the rotation of the table. In the water maze however, the alphalb(-/-) mice were unable to solve the task (acquisition and retention), whereas the control mice showed a good acquisition and retention behaviour. The knockout mice' incapacity to learn to reach the submerged platform was not due to an incapacity to swim, as they were as good as their control littermates to reach the platform when it was visible.

\section{LMG8}

OVEREXPRESSION OF MUTANT $\mathrm{Cu}, \mathrm{Zn}$ superoxide dismutase (SOD1) IN MICE MODEL OF FAMILIAL AMYOTROPHIC LATERAL SCLEROSIS (FALS) PRODUCES TASK-SPECIFIC EFFECTS ON LEARNING

V. Pietteur ${ }^{1,2}$, E. Paolucci ${ }^{2}$, S. Prisco ${ }^{2}$, A. Ferri $^{2}$, R. Gabbianelli ${ }^{2}$, and M. Ammassari-Teule ${ }^{1,2}$

${ }^{\prime}$ Istituto di Psicobiologia e Psicofarmacologia, Consiglio Nazionale delle Ricerche and ${ }^{2}$ IRCCS S. Lucia, Rome, Italy

We have carried out an investigation on possible consequences of the overexpression of mutant $\mathrm{Cu}, \mathrm{Zn}$ superoxide dismutase (SOD1) in a transgenic mice model for neurodegenerative disease familial amyotrophic lateral sclerosis (FALS). Three different groups of animals have been used in this series of experiments: (i) control mice (G1), expressing endogenous mouse $\mathrm{Cu}, \mathrm{Zn}$ superoxide dismutase only; (ii) transgenic mice (G2) overexpressing wild-type human SOD1 plus the endogenous mice SOD1 and (iii) transgenic mice (G3) overexpressing human mutant G93A SOD1, a protein found in patients affect with familial amyotrophic lateral sclerosis (FALS), plus the endogenous mice SOD1. Mice were first subjected to a five day active avoidance training. Subsequently the same animals were examined in a non 
associative task of object exploration followed by a test of reactivity to spatial change and to object change. Results of the first experiment indicated a strong performance deficit in mice overexpressing the mutant $\mathrm{Cu}, \mathrm{Zn}$ superoxide dismutase (G3) compared to mice belonging to the other groups (G1 and G2) among which no difference was found. Surprisingly, an inverted pattern of results was observed in the reactivity to spatial change since mice from G3 were found to fairly react to the new configuration of previously explored objects whereas mice from G1 and G2 did not. All mice, however, did react to the substitution of a previously explored object by a new one at the same location. Because (i) the reactivity to spatial change is highly controlled by the hippocampus and (ii) mice that react to spatial change generally show poor performance in active avoidance training, these results seem to indicate a paradoxical improvement of hippocampal function in mice carrying the G93A transgene relative to control mice or mice overexpressing wild type human SOD1.

\section{LMG9}

BEHAVIORAL AND BRAIN DOPAMINERGIC DYSFUNCTIONS IN PAH ENU-2 MICE: A PRECLINICAL MODEL OF PHENYLKETONURIA

T. Pascucci ${ }^{1,2 *}$, R. Ventura ${ }^{1,2}$, S. Cabib ${ }^{1,2}$, F. Cali $^{3}$, V. Romano ${ }^{3}$, and S. Puglisi-Allegra ${ }^{1}$

${ }^{\prime}$ Dept. Psychology, University of Rome "La Sapienza", ${ }^{2}$ Istituto di Psicobiologia e Psicofarmacologia (C. N. R.), Roma and, ${ }^{3}$ OASI Maria SS., IRCCS, Troina, Italy

Phenylketonuria (PKU), an autosomal recessive disorder, is caused by mutations in the gene encoding phenylalanine hydroxylase ( $\mathrm{Pah}$ ), the enzyme that converts phenylalanine (Phe) to tyrosine. The mutations result in the absence or reduced activity of Pah in the liver leading to elevated plasma Phe levels accompanied by decreased tyrosine levels. This genetic disorder, if untreated, can lead to brain damage and severe mental retardation. Moreover adolescents and young adults with early-treated but diet-discontinued PKU have been observed to present emotional difficulties, including anxiety, depression, poor social relationships, thought disorders, personality deviations and emotional liability $(3,4)$. Recently, using N-ethyl-N-nitrosurea (ENU) as a germline mutagen, a mouse model for human PKU has been developed (2) and a mutant mouse line has been produced, PAH ENU-2 (ENU-2) exhibiting phenotypes characteristic of classical PKU seen in humans. The experiments reported here evaluated brain dopamine (DA) functioning, cognitive impairment and emotional reactions in ENU-2 mice. Indeed a strict relationship between emotional reaction in aversive condition and brain DA neurotransmission has been recently envisaged (1). Homozygous (-/-) mice showed a dramatic impairment of learning of an active avoidance task. Moreover they exhibited a depression-like behavioural profile when tested in the forced swimming test. Homozygous (-/-) mice were also characterised by lower levels of DA, 3-4-dihydroxyphenylacetic acid (DOPAC), and homovanillic acid (HVA) in medial prefrontal cortex (pFC), ), nucleus accumbens (NAS) and caudatus-putamen (CP) in comparison with homozygous $(+/+)$ mice. Moreover, $(-/-)$ mice showed lower 5-HT and 5-HIAA levels in comparison with $(+/+)$ mice. These results demonstrate severe cognitive and emotional disturbances as 
well as major deficits in brain neurotransmitter functioning in ENU-2 mice, supporting the use of these animals as preclinical model of human phenylketonuria.

(1) Cabib \& Puglisi-Allegra (1996) Psychopharmacology, 128:331; (2) Shedlovsky et al., (1993) Genetics, 134:1205; (3) Waisbren, \& Levy (1991) J. Inherited Metabolic Disease 14:755; (4) Waisbren \& Zaff (1994) J. Inherited Metabolic Disease 17:584.

\title{
LMG10
}

\section{PHARMACOLOGICAL AND MOLECULAR EVIDENCES OF CAMP AND NF-kB DEPENDENT GENE EXPRESSION REGULATION IN LONG-TERM BUT NOT IN INTERMEDIATE-TERM MEMORY IN CHASMAGNATHUS}

\author{
A.G. Romano*, R. Freudenthal, F. Locatelli, G. Hermitte, and H. Maldonado
}

Laboratorio de Neurobiología de la Memoria, Dept. De Ciencias Biológicas, Facultad de Ciencias Exactas y Naturales, Universidad de Buenos Aires, Argentina

Protein synthesis and the activation of transcription factors have been shown to be a necessary step in long-term memory (LTM) consolidation both in invertebrates and vertebrates. In the Context-Stimulus Associative Learning Paradigm in the crab Chasmagnathus - like in other invertebrate models: the olfatory discrimination conditioning in Drosophila - spaced training has been shown to induce a longterm memory lasting at least for a week. This type of memory depends on de novo protein synthesis. On the contrary, massed training yields an intermediate-term memory lasting 2 or 3 days. Here we present evidences supporting that the formation of LTM, but not ITM, involves gene transcription regulation dependent on cAMP pathway and NF-kB transcription factors activation. In a first set of experiments, impairment of LTM but not ITM was shown when the protein synthesis inhibitor cycloheximide was administrated. Similarly, the injection of two cAMP analogues, either specific activator or inhibitor of PKA, affected LTM but not ITM. Futhermore, NF-kB activity is enhanced after spaced but not after massed training. This activation showed two phases, a rapid and transient activation immediately after training that dropped after $3 \mathrm{hs}$ and a second phase that peaked at $6 \mathrm{hs}$ after training and reached the basal level at 24hours. Thus, gene expression dependent on cAMP and NF-kB pathways is thought to be involved in LTM formation but not in an intermediate form of memory. 

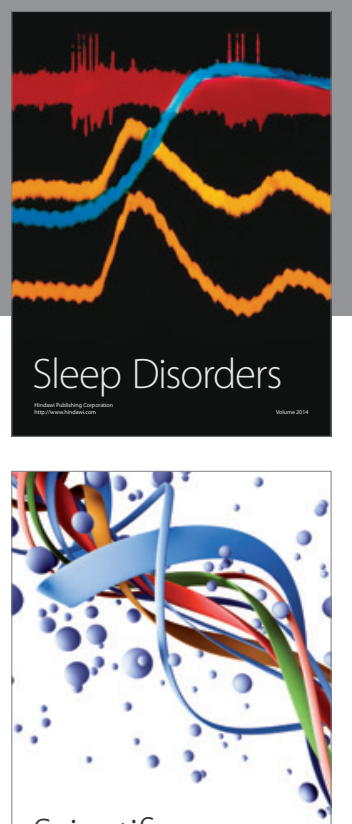

Scientifica
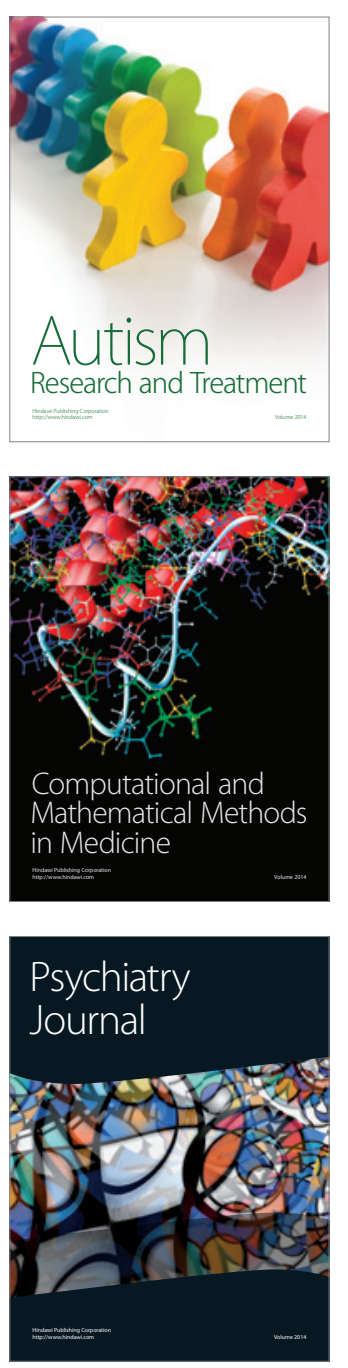
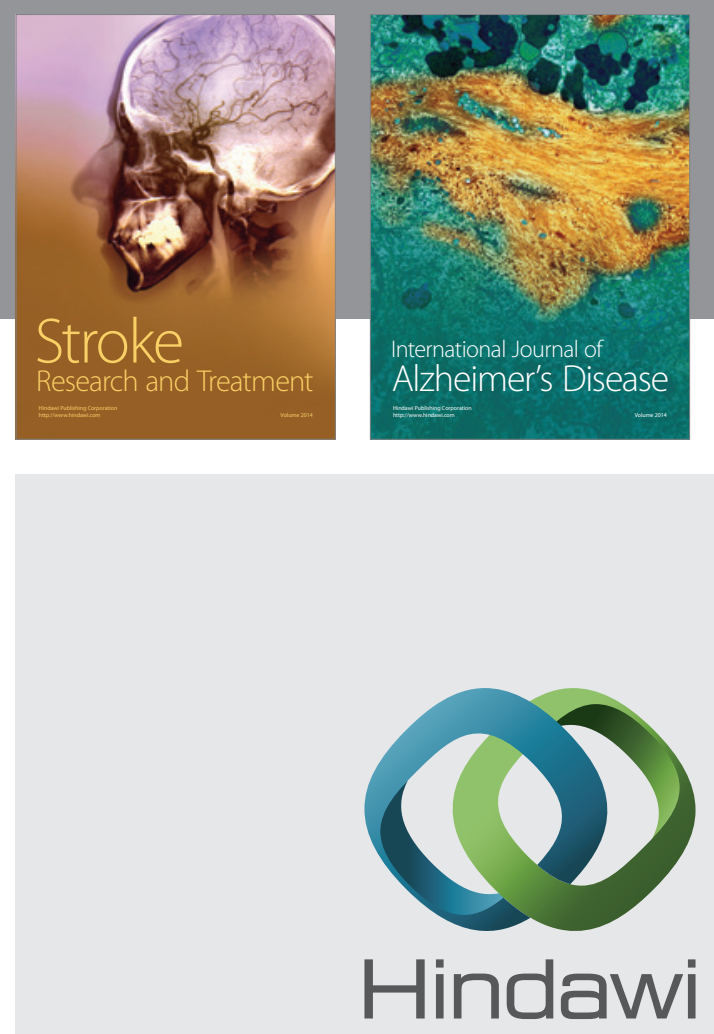

Submit your manuscripts at

http://www.hindawi.com
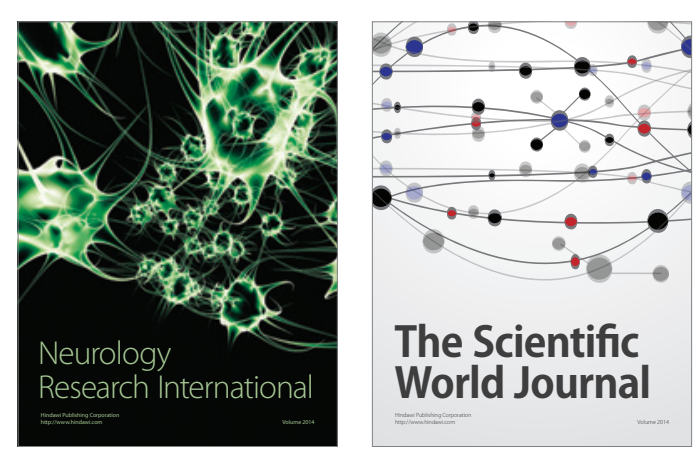

The Scientific World Journal

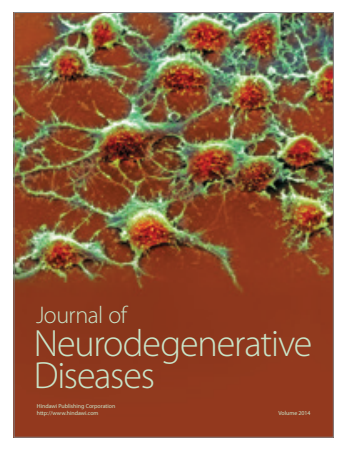

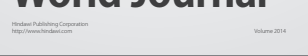

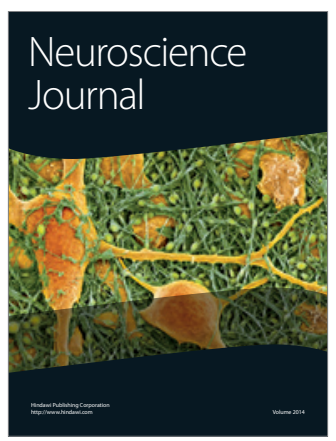

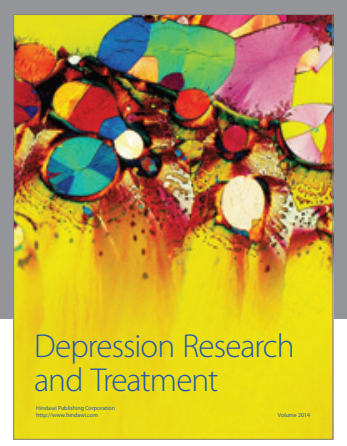
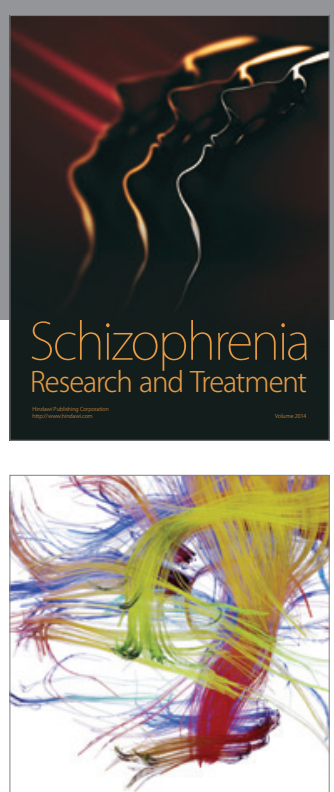

Brain Science

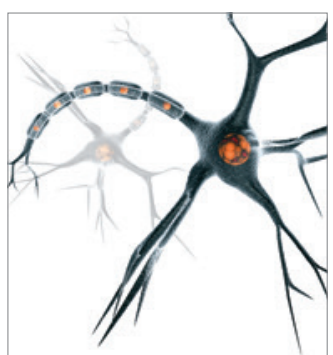

Neural Plasticity
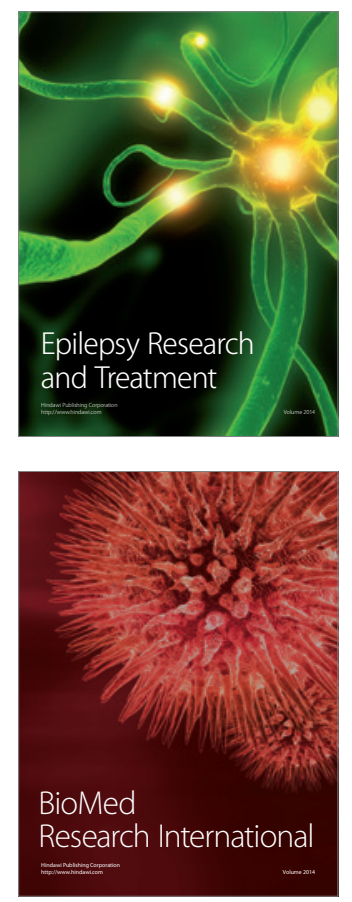

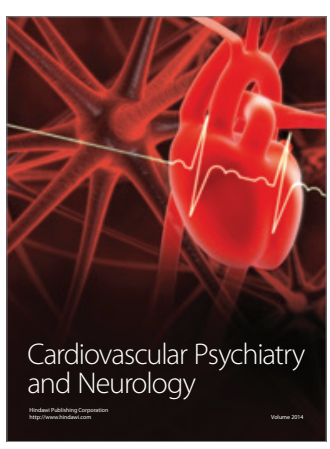

Parkinson's

Disease
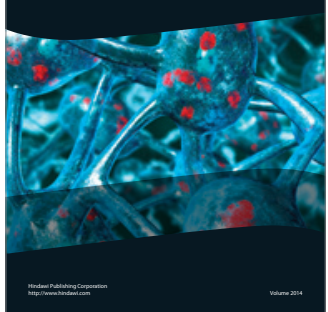